\title{
Analysis of Proprioception of Hip Joint in Total Hip Arthroplasty
}

\author{
Hitoshi Onishi' ${ }^{1}$, Satoshi Nagoya ${ }^{2}$, Tsuneo Takebayashi' ${ }^{1}$, Toshihiko Yamashita ${ }^{1}$ \\ ${ }^{1}$ Department of Orthopaedic Surgery, School of Medicine, Sapporo Medical University, Sapporo, Japan \\ ${ }^{2}$ Department of Musculoskeletal Biomechanics and Surgical Development, School of Medicine, Sapporo Medical University, \\ Sapporo, Japan \\ Email: onishi12341979@yahoo.co.jp
}

How to cite this paper: Onishi, H., Nagoya, S., Takebayashi, T. and Yamashita, T. (2017) Analysis of Proprioception of Hip Joint in Total Hip Arthroplasty. Open Journal of Orthopedics, 7, 53-62. https://doi.org/10.4236/ojo.2017.72007

Received: December 19, 2016

Accepted: February 5, 2017

Published: February 8, 2017

Copyright $\odot 2017$ by authors and Scientific Research Publishing Inc. This work is licensed under the Creative Commons Attribution International License (CC BY 4.0).

http://creativecommons.org/licenses/by/4.0/

\begin{abstract}
Introduction: The instability of the joint is classified roughly into mechanical and functional. It is reported that the postoperative dislocation often occurs by the posterior approach of the THA and may be caused by functionality instability due to the injury of the periarticular soft tissue. We analyzed the joint position sense of the hip according to an approach of the THA and examined effect to give postoperative dislocation. Materials \& Methods: 92 patients (184 hip joints) who received THA in our hospital were selected in the study. Cases in which position sensation measurements were insufficient were excluded. As for the classification, the posterior approach (PL groups) was 39 hips, anterolateral approach (AL groups) was 30 hips, and control group was 37 hips. Results: There was no significant difference between the AL group and PL group in the absolute reproduction angle error score (ARAES). The relative reproduction angle error scores (RRAES) for passive internal and external rotations and active internal rotation were significantly lower in the $\mathrm{AL}$ and control groups than the PL group. Discussion: This study was suggested that the hip joint position sense in the AL group was better retained for the preservation of the soft tissue.
\end{abstract}

\section{Keywords}

Hip Joint Position Sense, Total Hip Arthroplasty, Proprioception

\section{Introduction}

Recently, total hip arthroplasty (THA) has been performed for patients with various types of hip disorders. They included osteoarthritis, rheumatoid arthritis, Perthes deformity, and osteonecrosis of the femoral head. Although THA is an effective procedure to achieve pain relief and restore the hip functions, dislocation is apprehensive complication after THA, which is the most common 
cause of THA revisions. Many factors contributed dislocation of THA, such as vertical implantation of acetabular components, reduced offset of the hip, inadequate combined anteversion and reduced tension of soft tissue around the hip joint. It was reported that it often occurs in the first year after surgery when the posterolateral approach (PL) is used, whereas it occurs later with the anterolateral approach (AL). When appropriate implantation of prosthesis is accomplished, instability should be focused in terms of dislocation after operation. There are two types of joint instability: one is mechanical instability due to the disruption of anatomical structures of the hip joints and the other one is functional instability due to insufficient proprioception (joint position sense and kinesthetic sense) [1] [2]. Although mechanical instability [3] may be important as contributing factors of dislocation, the fact that some dislocations occur more than 10 years after operation indicates that the cause of dislocation is not only mechanical factors but also functional instability due to disruption of joint proprioception [4] [5]. Therefore, we analyzed the joint position sense in patients treated by THA through different approach and examined if there were any differences between the two surgical approaches in the hip joint position sense.

\section{Materials and Methods}

Of the patients who received THA in our hospital from June 1991 to July 2014, 92 outpatients (184 hip joints) who were treated from April 2012 to October 2014 were selected and enrolled in the study. The exclusion criteria could not understand method for measurement and did it with the patients whom the measurement was not able to conclude or the patients with the dementia. Of these, 12 were male and 80 were female. The mean age was 63.1 years (range, 22 - 84 years), and the mean postoperative follow-up was 65.8 months (range, 3 312 months). Healthy contralateral hip joints without radiographic abnormality were assigned to the control group. We performed THA through two types of surgical approaches. One of approach was anterolateral modified Watson jones's approach (AL group) and the other was posterolateral approach (PL group). The AL group included 30 hips, the PL group included 39 hips, and the control group included 37 hips. In the AL group, the mean patient age was $63 \pm 11.3$ years, the mean postoperative follow-up period was $20.9 \pm 17.4$ months, and four and 25 patients were male and female, respectively. Fourteen right joints and 16 left joints were used in the study. In the PL group, the mean patient age was $63.4 \pm 10.6$ years, the mean postoperative follow-up period was $107.2 \pm 80.2$ months, and six and 23 patients were male and female, respectively. Twenty right joints and 19 joints were included in the study. In the control group, the mean patient age was $63.8 \pm 11.9$ years, four patients were male, and 33 were female. Nineteen right joints and 18 left joints were analyzed (Table 1). During the examination of patients in the AL group for surgery, osteoarthritis of the hip was diagnosed in 20 joints, osteonecrosis of femoral head was observed in eight cases, and rheumatoid arthritis occurred in two joints. In the PL group, osteoarthritis of the hip was diagnosed in 28 hip joints and osteonecrosis of the 
femoral head occurred in 11 cases. The joints in the control group had no joint space narrowing, osteoarthritis of the femoral head, or acetabular roof. We examined items from the Visual Analog Scale (VAS), University of California Los Angeles (UCLA) activity-level rating, Oxford hip score, and Harris hip score and were used to measure hip joint position sense using the method described below.

An examiner measured joint position sense manually using a measurement device (Figure 1). Visual confirmation of the angles was made using a goniometer, which could measure the same angle of the lower extremities. Patients were placed in the semi-Fowler's position and asked to close their eyes during measurements. The hip joints were rotated internally and externally to $20^{\circ}$, held in position for $5 \mathrm{~s}$, and the patients were then asked to memorize the angle. The joints were returned to $0^{\circ}$ (initial position), and the patients were asked to repeat the movement and declare when they had reached the memorized angle. The actual measurements were used as the angular repositioning values. Active and passive movements were used to measure the angles. The rotation speed was set at $2 \%$ for passive movements, and measurements were performed three times.

Table 1. Demographics of the study groups.

\begin{tabular}{cccc}
\hline Group & AL & PL & Control \\
\hline Number & 30 & 39 & 37 \\
Age (years) & $63.0 \pm 11.3$ & $63.4 \pm 10.6$ & $63.8 \pm 11.9$ \\
Duration of disease & $30.4 \pm 12.2$ & $35.2 \pm 15.3$ & $4 / 33$ \\
Months from surgery & $20.9 \pm 17.4$ & $107.2 \pm 80.2$ & $19 / 18$ \\
Sex (male/female) & $4 / 25$ & $6 / 23$ & \\
Dominant (Rt/Lt) & $14 / 16$ & $20 / 19$ & \\
Osteoarthritis & 20 & 28 & \\
Oiease & 8 & & \\
\hline
\end{tabular}

Values are mean \pm SD (standard deviation)

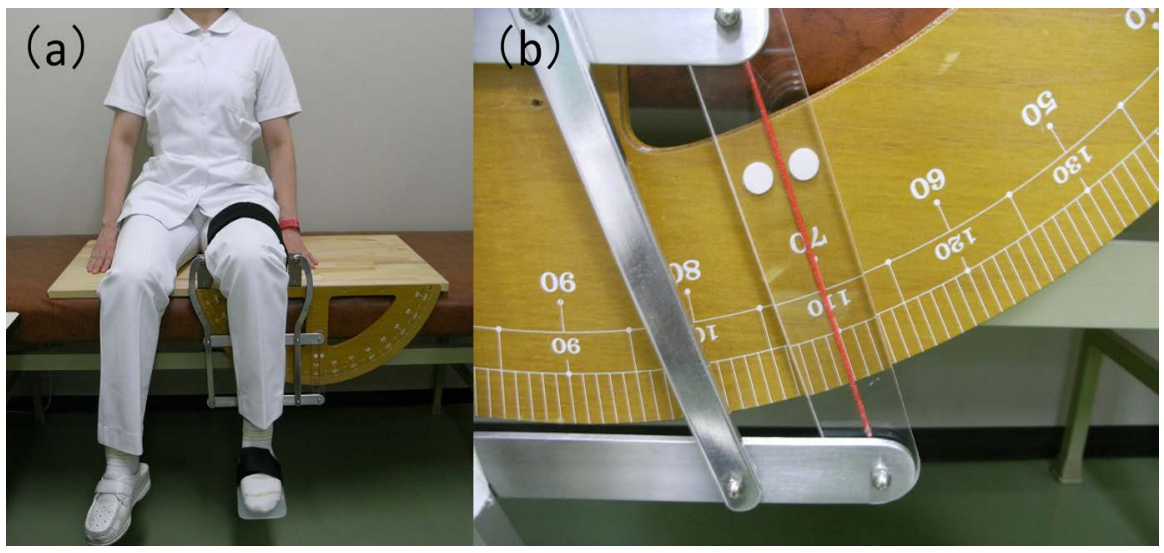

Figure 1. Original goniometter for the present cohort study. (a) Using a thigh corset, we relieve a friction between the thigh and skin that may occur by rotational movement. (b) The view of the joint angle measurement. 
The absolute reproduced angle error score (ARAES) was defined as the absolute value of the score attracted $20^{\circ}$ from a reproduced angle. In addition, the relative reproduced angle error score (RRAES) was defined as the difference between the maximum and minimum values of the reproduced angle. Soft Statcel3 (OMS publishing Inc. Japan) was used for statistical analyses after approval by our institution's statistical official. Mann-Whitney U tests, Student's $t$-tests, and Kruskal-Wallis tests were used for statistical analyses.

\section{Results}

There were no statistically significant differences between the AL group, PL group, and control group in the assessment items for VAS, UCLA activity level rating, Oxford hip score, and Harris hip score (Table 2). Also there were no significant differences in the active or passive angular repositioning of external or internal $20^{\circ}$ rotations among the three groups (Table 3 ).

The active internal rotation ARAES was $3.40^{\circ} \pm 2.11^{\circ}, 4.03^{\circ} \pm 2.57^{\circ}$, and $2.89^{\circ}$ $\pm 1.83^{\circ}$ in the AL group, PL group, and control group, respectively; there were no significant differences among groups. The active external rotation ARAES was $2.48^{\circ} \pm 1.95^{\circ}, 3.14^{\circ} \pm 1.68^{\circ}$, and $2.76^{\circ} \pm 2.16^{\circ}$ in the AL group, PL group, and control group, respectively; there was a significant difference between the AL group and PL group (Figure 2, Table 4). The passive internal rotation ARAES was $2.56^{\circ} \pm 1.38^{\circ}, 2.68^{\circ} \pm 1.49^{\circ}$, and $2.13^{\circ} \pm 1.70^{\circ}$ in the AL group, PL group, and control group, respectively, compared with respective passive external rotation ARAES of $2.40^{\circ} \pm 1.74^{\circ}, 2.64^{\circ} \pm 1.60^{\circ}$, and $2.17^{\circ} \pm 1.70^{\circ}$. There were no significant differences in these errors among the groups (Figure 3, Table 4).

Table 2. Postoperative clinical evaluation.

\begin{tabular}{ccccc}
\hline Group & AL & PL & Control & $p$ value \\
\hline VAS & $6.9 \pm 8.8$ & $12.5 \pm 21.3$ & $12.2 \pm 21.6$ & 0.73 \\
UCLA Score & $4.9 \pm 1.6$ & $5.2 \pm 1.4$ & $5.1 \pm 1.4$ & 0.71 \\
Oxford Hip Score & $20.7 \pm 6.8$ & $22.5 \pm 10.6$ & $22.0 \pm 8.5$ & 0.89 \\
Harris Hip Score & $86.7 \pm 10.7$ & $84.0 \pm 13.4$ & $87.1 \pm 10.3$ & 0.73 \\
\hline
\end{tabular}

Values are mean \pm SD (standard deviation); VAS: Visual Analog Scale; UCLA Score: University of California Los Angeles activity-level rating.

Table 3. Repositioning of rotation angle.

\begin{tabular}{cccccc}
\hline \multirow{2}{*}{ Group } & & $\mathrm{AL}$ & $\mathrm{PL}$ & Control & $p$ value \\
\hline \multirow{3}{*}{ Active motion } & Internal arc & $21.0 \pm 3.9$ & $21.7 \pm 4.4$ & $21.5 \pm 3.1$ & 0.711 \\
& External arc & $19.5 \pm 3.1$ & $18.9 \pm 3.3$ & $20.7 \pm 3.4$ & 0.063 \\
& Total arc & $40.5 \pm 5.2$ & $40.5 \pm 6.1$ & $42.3 \pm 4.7$ & 0.345 \\
\hline \multirow{3}{*}{ Passive motion } & Internal arc & $19.3 \pm 2.8$ & $19.7 \pm 2.9$ & $19.5 \pm 2.6$ & 0.912 \\
& External arc & $19.4 \pm 2.9$ & $18.8 \pm 2.8$ & $19.7 \pm 2.7$ & 0.387 \\
& Total arc & $38.8 \pm 4.7$ & $38.5 \pm 4.6$ & $39.2 \pm 4.3$ & 0.794 \\
\hline
\end{tabular}

Values are mean \pm SD (standard deviation). 
(a)

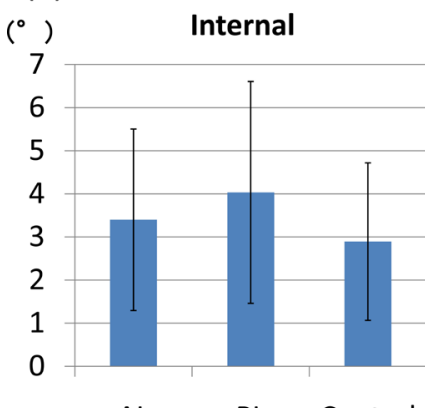

AL PL Control (b)

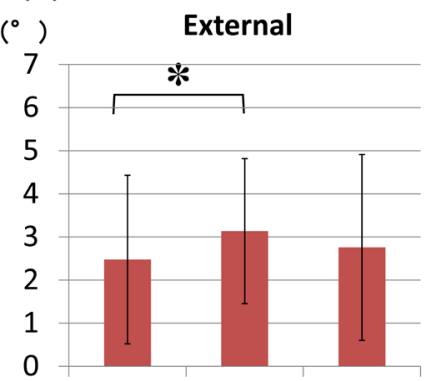

AL PL Control

$*: p<0.05$

Figure 2. Absolute reproduction angle error score (ARAES) of the active repositioning tasks Bar graph plots score of ARAES for AL, PL and control groups in the internal (a) and external (b) hip rotation tasks.

(a)

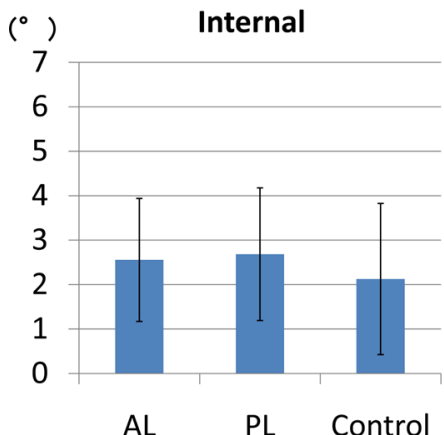

(b)

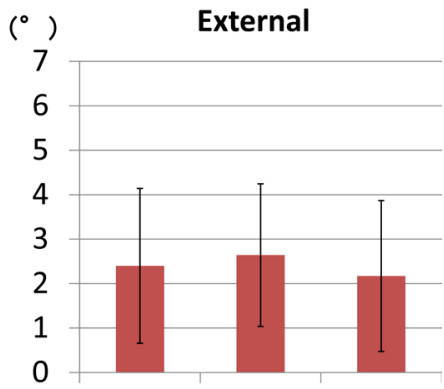

$\mathrm{AL}$
PL Control

Figure 3. Absolute reproduction angle error score (ARAES) of the passive repositioning tasks Bar graph plots score of ARAES for AL, PL and control groups in the internal (a) and external (b) hip rotation tasks.

Table 4. ARAES of measured value.

\begin{tabular}{|c|c|c|c|c|c|c|c|}
\hline & & \multicolumn{3}{|c|}{ Internal } & \multicolumn{3}{|c|}{ External } \\
\hline & & $\mathrm{AL}$ & PL & Control & $\mathrm{AL}$ & PL & Control \\
\hline \multirow{3}{*}{ Active } & Mean & 3.40 & 4.03 & 2.89 & 2.48 & 3.14 & 2.76 \\
\hline & $\mathrm{SD}$ & 2.11 & 2.57 & 1.83 & 1.95 & 1.68 & 2.16 \\
\hline & & $\mathrm{AL}$ & PL & Control & $\mathrm{AL}$ & PL & Control \\
\hline \multirow{2}{*}{ Passive } & Mean & 2.56 & 2.68 & 2.13 & 2.40 & 2.64 & 2.17 \\
\hline & SD & 1.38 & 1.49 & 1.70 & 1.74 & 1.60 & 1.70 \\
\hline
\end{tabular}

Note: $\mathrm{SD}=$ standard deviation.

The active internal rotation RRAES was $1.77^{\circ} \pm 1.02^{\circ}, 3.08^{\circ} \pm 1.70^{\circ}$, and $2.30^{\circ}$ $\pm 1.66^{\circ}$ in the $\mathrm{AL}$ group, PL group, and control group, respectively; there were significant differences between the AL and PL groups and the PL and control groups. The active external rotation RRAES was $2.27^{\circ} \pm 1.18^{\circ}, 2.92^{\circ} \pm 1.95^{\circ}$, and $2.81^{\circ} \pm 1.50^{\circ}$ in the AL group, PL group, and control group, respectively, and there were no significant differences among the groups (Figure 4, Table 5).

The passive internal rotation RRAES for the groups was $2.33^{\circ} \pm 1.14^{\circ}, 3.26^{\circ} \pm$ 
$1.84^{\circ}$, and $1.76^{\circ} \pm 1.00^{\circ}$ in the $\mathrm{AL}$ group, PL group, and control group, respectively, compared with the respective passive external rotation RRAES of $2.00^{\circ} \pm$ $1.24^{\circ}, 2.74^{\circ} \pm 1.53^{\circ}$, and $1.86^{\circ} \pm 1.21^{\circ}$. There were significant differences between the AL and PL groups and between the PL and control groups (Figure 5, Table 5).

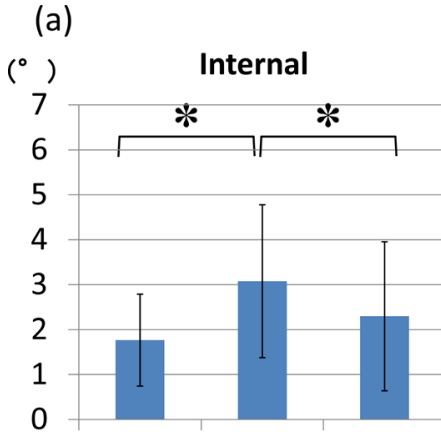

$\mathrm{AL}$ (b)

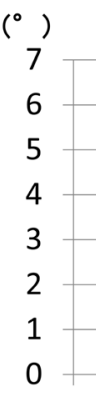

$A L$
External

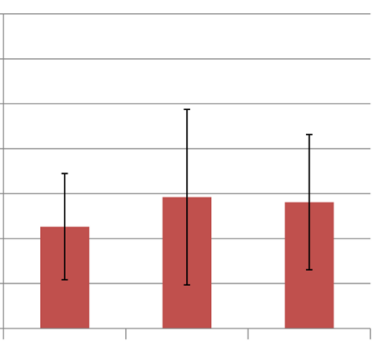

PL Control

$*: p<0.05$

Figure 4. Relative reproduction angle error score (RRAES) of the active repositioning tasks Bar graph plots score of RRAES for AL, PL and control groups in the internal (a) and external (b) hip rotation tasks.

(a)

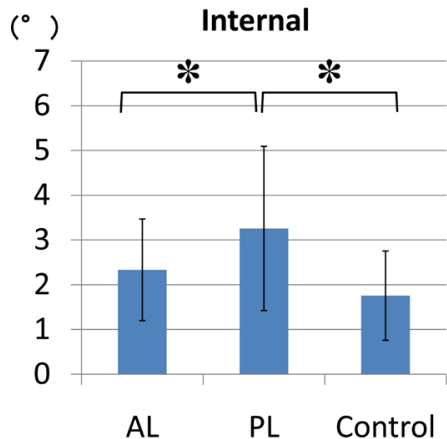

(b)

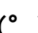

$\left({ }^{\circ}\right)$

0

$\mathrm{AL}$

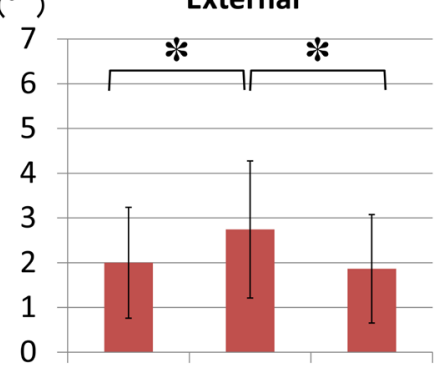

\section{External}

PL Control

$*: p<0.05$

Figure 5. Relative reproduction angle error score (RRAES) of the passive repositioning tasks Bar graph plots score of RRAES for AL, PL and control groups in the internal (a) and external (b) hip rotation tasks.

Table 5. RRAES of measured value.

\begin{tabular}{cccccccc}
\hline & & \multicolumn{3}{c}{ Internal } & \multicolumn{3}{c}{ External } \\
\hline \multirow{2}{*}{ Active } & Mean & 1.77 & 3.08 & 2.30 & 2.27 & 2.92 & 2.81 \\
& SD & 1.02 & 1.70 & 1.66 & 1.18 & 1.95 & 1.50 \\
\hline \multirow{2}{*}{ Passive } & Mean & 2.33 & 3.26 & 1.76 & 2.00 & 2.74 & 1.86 \\
& SD & 1.14 & 1.84 & 1.00 & 1.24 & 1.53 & 1.21 \\
\hline
\end{tabular}

Note: $\mathrm{SD}=$ standard deviation. 


\section{Discussion}

In recent years, the anterolateral approach has used for THA to achieve invasiveness to the soft tissues around the hip joint, which has possibly led to a decrease dislocations after operation. In 1999, Emile et al. reported that the rate of post-THA dislocation was $3.9 \%$, compared with only $0.7 \%$ by Mirza et al. in 2014 [6] [7]. Other studies have reported differences in the postoperative dislocation depending on the approach used. Berry et al. reported a rate of $3.1 \%$ for the AL method and $6.9 \%$ for the PL method. In addition, Jeya et al. reported a rate of $1.7 \%$ for the AL method and 2.3\% for the PL method, whereas Masoins et al. reported rates of $2.18 \%$ and $3.23 \%$ for the AL and PL methods, respectively [8] [9] [10]. Furthermore, Marcel et al. reported a six-times higher incidence of dislocations after the use of the PL method compared with the AL method [11].

Previous reports demonstrated that the mechanoreceptor in the hip joint has little effect on position sensation, and that the periarticular tissue plays a role [12] [13] [14]. However, Moraes et al. reported that mechanoreceptors controlling the peculiar position sensation of the hip joint are present in the joint capsule (round ligament and acetabular labrum) [15]. When joint capsule after the THA is kept although there is difference in the approach, there is the report to influence position sensation [16]. It is possible that the AL approach conserves the posterior soft tissues better than the PL approach, and that the remaining proprioception is involved in joint stability.

A comparative study of hip joint position sense by Pickard et al. reported angular internal and external rotation measurements in elderly and young patients [17]. The error of internal rotation was small and thus relatively accurate; however, active movements produced more accurate angles than passive movements. Moreover, age was not related to the accuracy of angular repositioning. In a study measuring the position sense of active and passive movements in healthy adults aged 18 - 30 years, Benjaminse et al. reported that passive movements were more accurate [18]. A study comparing THA and hip resurfacing by Larkin et al., reported that, compared with THA, hip resurfacing tended to ameliorate proprioception, but not in elderly subjects [19]. Based on these previous studies, it is unclear whether active or passive movement is more accurate; the correlation with age is also unknown. Various measurement methods were used in these studies, and therefore, no consensus has been reached.

The peripheral muscles in the hip joint move during its active movement. Thus, proprioception of the posterior soft tissues and antagonist muscles is needed to control these movements. In contrast, during passive movement the peripheral muscles of the hip joint do not move; therefore, only proprioception of the posterior soft tissues is used to control the dislocation position of the hip joint. In the present study, the angular error scores for both passive and active movements were similar in the AL group and the control group. In addition, there was a significant difference between the AL and PL groups. Therefore, these results suggest that the use of the AL approach conserves the posterior joint capsule that controls both active and passive movements, thereby conserv- 
ing proprioception in patients.

In the current study, we measured the rotational movement angles of the hip joints in the AL, PL, and the control groups. There were no significant differences in the scores of any patient assessment items between the AL and PL groups. However, there were significant differences in the external rotation direction of the active measurement in ARAES between the AL and PL groups. There was no significant difference between the AL group and the control group. In contrast, there were no significant differences in the internal rotation direction of the active and passive measurements in ARAES among the three groups.

In contrast, significant differences were observed between the AL and PL groups and the PL and control groups in the internal rotation direction of the active measurement in RRAES; specifically, the PL group was significantly higher than the other two groups. Furthermore, there were significant differences between the AL and PL groups and the PL and the control groups in the internal and external rotation direction of the passive measurement in RRAES; the RRAES of the PL group was significantly larger than those of the AL and control groups. However, the RRAES was similar in the AL and control groups.

ARAES shows the size of the deviation from a defined point, but there were no significant differences among the groups. In contrast, RRAES shows a difference in the reproduction angle that a subject recognizes. The PL group recognized other two groups and significant difference, but there was no significant difference between the AL and control groups. Therefore, we recognize neighborhood of the set-point after THA, but a drop of the endurance of the recognition is guessed in the PL group where a backward soft tissue received the invasion. In addition, the hip joint position sensation of the AL group is likely similar to that in the control group.

The limitations of the present study are as follows. First, the effects of gravity and skin sensation could not be eliminated. Nevertheless, patient-related differences can be controlled by making adjustments to the device. When performing the same angle repositioning of internal and external rotations in post-THA patients, it is difficult to use positions other than the sitting position. Although it is possible to place young patients in the lateral decubitus position, it is difficult to do the same for elderly patients. Furthermore, evaluating a range of rotational motions $>20^{\circ}$ was difficult because of the possibility of dislocation.

The angular error scores of passive internal and external rotations and active internal rotation were significantly smaller in the AL group compared with the PL group. Therefore, these results suggest that proprioception was conserved well in the AL group compared with the PL group.

\section{Conclusion}

We compared post-THA joint rotation position sense in 30 joints in the AL group, 39 in the PL group, and 37 in the control group. There was no significant difference between the AL group and PL group in the absolute reproduction angle error score (ARAES). The relative reproduction angle error scores (RRAES) 
for passive internal and external rotations and active internal rotation were significantly lower in the AL group than the PL group. A comparison of the results in the PL and AL groups suggests that joint position sense was conserved in the AL group compared to the PL group.

\section{References}

[1] Hubbard, T.J., Kramer, L.C., Denegar, C.R. and Hertel, J. (2007) Correlations among Multiple Measures of Functional and Mechanical Instability in Subjects with Chronic Ankle Instability. Journal of Athletic Training, 42, 361-366.

[2] Hiller, C.E., Kilbreath, S.L. and Refshauge, K.M. (2011) Chronic Ankle Instability: Evolution of the Model. Journal of Athletic Training, 46, 133-141. https://doi.org/10.4085/1062-6050-46.2.133

[3] Blom, A.W., Rogers, M., Taylor, A.H., Pattison, G., Whitehouse, S. and Bannister, G.C. (2008) Dislocation Following Total Hip Replacement: The Avon Orthopaedic Centre Experience. Annals of the Royal College of Surgeons of England, 90, 658662. https://doi.org/10.1308/003588408X318156

[4] Knoch, M.V., Berry, D.J., Harmsen, W.S. and Morrey, B.F. (2002) Late Dislocation after Total Hip Arthroplasty. Journal of Bone and Joint Surgery. American Volume, 84, 1949-1953. https://doi.org/10.2106/00004623-200211000-00007

[5] Lidder, S., Ranawat, V.S., Ranawat, N.S. and Thomas, T.L. (2009) Chronic Asymptomatic Dislocation of a Total Hip Replacement: A Case Report. Journal of Medical Case Reports, 3, 8956. https://doi.org/10.4076/1752-1947-3-8956

[6] Li, E., Meding, J.B., Ritter, M.A., Keating, E.M. and Faris, P.M. (1999) The Natural History of a Posteriorly Dislocated Total Hip Replacement. Journal of Arthroplasty, 14, 964-968. https://doi.org/10.1016/S0883-5403(99)90011-6

[7] Mirza, A.J., Lombardi Jr., A.V., Morris, M.J. and Berend, K.R. (2014) A Mini-Anterior Approach to the Hip for Total Joint Replacement: Optimising Results. Bone \& Joint Journal, 96B, 32-35. https://doi.org/10.1302/0301-620X.96B11.34348

[8] Berry, D.J., von Knoch, M., Schleck, C.D. and Harmsen, W.S. (2005) Effect of Femoral Head Diameter and Operative Approach on Risk of Dislocation after Primary Total Hip Arthroplasty. Journal of Bone and Joint Surgery. American Volume, 87, 2456-2463.

[9] Palan, J., Beard, D.J., Murray, D.W., Andrew, J.G. and Nolan, J. (2009) Which Approach for Total Hip Arthroplasty Anterolateral or Posterior? Clinical Orthopaedics and Related Research, 467, 473-477. https://doi.org/10.1007/s11999-008-0560-5

[10] Masonis, J.L. and Bourne, R.B. (2002) Surgical Approach, Abductor Function, and Total Hip Arthroplasty Dislocation. Clinical Orthopaedics and Related Research, 405, 46-53. https://doi.org/10.1097/00003086-200212000-00006

[11] Dudda, M., Gueleryuez, A., Gautier, E., Busato, A. and Roeder, C. (2010) Risk Factors for Early Dislocation after Total Hip Arthroplasty: A Matched Case-Control Study. Journal of Orthopaedic Surgery, 18, 179-183. https://doi.org/10.1177/230949901001800209

[12] Grigg, P., Finerman, G.A. and Riley, L.H. (1973) Joint-Position Sense after Total Hip Replacement. The Journal of Bone and Joint Surgery, 55, 1016-1025. https://doi.org/10.2106/00004623-197355050-00010

[13] Ishii, Y., Tojo, T., Terajima, K., Terashima, S. and Bechtold, J.E. (1999) Intracapsular Components Do Not Change Hip Proprioception. The Journal of Bone and Joint Surgery, 81, 345-348. https://doi.org/10.1302/0301-620X.81B2.9104 
[14] Karanjia, P.N. and Ferguson, J.H. (1983) Passive Joint Position Sense after Total Hip Replacement Surgery. Annals of Neurology, 13, 654-657. https://doi.org/10.1002/ana.410130612

[15] Moraes, M.R.B., Cavalcante, M.L.C., Leite, J.A.D., Macedo, J.N., Sampaio, M.L.B., Jamacaru, V.F. and Santan, M.G. (2011) The Characteristics of the Mechanoreceptors of the Hip with Arthrosis. Journal of Orthopaedic Surgery and Research, 6, 58.

[16] Holnapy, G. and Kiss, R.M. (2013) Impact of the Method of Exposure in Total Hip Arthroplasty on Balancing Ability in Response to Sudden Unidirectional Perturbation in the First Six Months of the Postoperative Period. Journal of Electromyography and Kinesiology, 23, 727-733. https://doi.org/10.1016/j.jelekin.2013.01.005

[17] Pickard, C.M., Sullivan, P.E., Allison, G.T. and Singer, K.P. (2003) Is There a Difference in Hip Joint Position Sense between Young and Older Groups? Journals of Gerontology Series A: Biological Sciences and Medical Sciences, 58, M631-M635. https://doi.org/10.1093/gerona/58.7.M631

[18] Benjaminse, A., Sell, T.C., Abt, J.P., House, A.J. and Lephart, S.M. (2009) Reliability and Precision of Hip Proprioception Methods in Healthy Individuals. Clinical Journal of Sport Medicine, 19, 457-463. https://doi.org/10.1097/JSM.0b013e3181bcb155

[19] Larkin, B., Nyazee, H., Motley, J., Nunley, R.M., Clohisy, J.C. and Barrack, R.L. (2013) Hip Resurfacing Does Not Improve Proprioception Compared with THA. Clinical Orthopaedics and Related Research, 471, 3387-3388. https://doi.org/10.1007/s11999-013-3210-5

Submit or recommend next manuscript to SCIRP and we will provide best service for you:

Accepting pre-submission inquiries through Email, Facebook, LinkedIn, Twitter, etc. A wide selection of journals (inclusive of 9 subjects, more than 200 journals) Providing 24-hour high-quality service User-friendly online submission system Fair and swift peer-review system Efficient typesetting and proofreading procedure Display of the result of downloads and visits, as well as the number of cited articles Maximum dissemination of your research work

Submit your manuscript at: http://papersubmission.scirp.org/

Or contact ojo@scirp.org 